\title{
Digital Surveillance Through an Online Decision Support Tool for COVID-19 Over One Year of the Pandemic in Italy: Observational Study
}

Alberto Eugenio Tozzi ${ }^{1}, \mathrm{MD}$; Francesco Gesualdo ${ }^{1}, \mathrm{MD}$; Emanuele Urbani ${ }^{2}$, MD; Alessandro Sbenaglia ${ }^{2}, \mathrm{MSc}$ Roberto Ascione ${ }^{3}$, MSc; Nicola Procopio ${ }^{3}$, MSc; Ileana Croci $^{1}$, DStat; Caterina Rizzo ${ }^{4}, \mathrm{MD}$

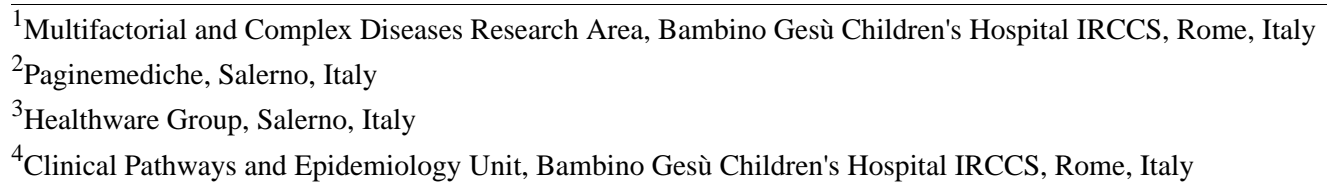

\section{Corresponding Author:}

Francesco Gesualdo, MD

Multifactorial and Complex Diseases Research Area

Bambino Gesù Children's Hospital IRCCS

Piazza S. Onofrio, 4

Rome, 00165

Italy

Phone: 393388437474

Email: francesco.gesualdo@opbg.net

\section{Abstract}

Background: Italy has experienced severe consequences (ie, hospitalizations and deaths) during the COVID-19 pandemic. Online decision support systems (DSS) and self-triage applications have been used in several settings to supplement health authority recommendations to prevent and manage COVID-19. A digital Italian health tech startup, Paginemediche, developed a noncommercial, online DSS with a chat user interface to assist individuals in Italy manage their potential exposure to COVID-19 and interpret their symptoms since early in the pandemic.

Objective: This study aimed to compare the trend in online DSS sessions with that of COVID-19 cases reported by the national health surveillance system in Italy, from February 2020 to March 2021.

Methods: We compared the number of sessions by users with a COVID-19-positive contact and users with COVID-19-compatible symptoms with the number of cases reported by the national surveillance system. To calculate the distance between the time series, we used the dynamic time warping algorithm. We applied Symbolic Aggregate approXimation (SAX) encoding to the time series in 1-week periods. We calculated the Hamming distance between the SAX strings. We shifted time series of online DSS sessions 1 week ahead. We measured the improvement in Hamming distance to verify the hypothesis that online DSS sessions anticipate the trends in cases reported to the official surveillance system.

Results: We analyzed 75,557 sessions in the online DSS; 65,207 were sessions by symptomatic users, while 19,062 were by contacts of individuals with COVID-19. The highest number of online DSS sessions was recorded early in the pandemic. Second and third peaks were observed in October 2020 and March 2021, respectively, preceding the surge in notified COVID-19 cases by approximately 1 week. The distance between sessions by users with COVID-19 contacts and reported cases calculated by dynamic time warping was 61.23 ; the distance between sessions by symptomatic users was 93.72 . The time series of users with a COVID-19 contact was more consistent with the trend in confirmed cases. With the 1-week shift, the Hamming distance between the time series of sessions by users with a COVID-19 contact and reported cases improved from 0.49 to 0.46 . We repeated the analysis, restricting the time window to between July 2020 and December 2020. The corresponding Hamming distance was 0.16 before and improved to 0.08 after the time shift.

Conclusions: Temporal trends in the number of online COVID-19 DSS sessions may precede the trend in reported COVID-19 cases through traditional surveillance. The trends in sessions by users with a contact with COVID-19 may better predict reported cases of COVID-19 than sessions by symptomatic users. Data from online DSS may represent a useful supplement to traditional surveillance and support the identification of early warning signals in the COVID-19 pandemic. 
(J Med Internet Res 2021;23(8):e29556) doi: 10.2196/29556

\section{KEYWORDS}

COVID-19; public health; surveillance; digital surveillance; internet; online decision support system; decision support; support; online tool; Italy; observational

\section{Introduction}

As of March 2021, the World Health Organization had estimated that almost 120 million cases of COVID-19 had occurred worldwide, with more than 2.5 million deaths [1]. The first cases of COVID-19 in Europe date back to January 2020, with the first 2 cases identified in Italy on January 31 in 2 Chinese tourists [2]. Since then, Italy has been severely hit by the pandemic [3]. As in other countries, from the earliest phases of the pandemic, the Italian Ministry of Health developed an information campaign with recommendations for managing contacts of SARS-CoV-2-positive individuals and information on symptoms that could suggest a possible diagnosis of COVID-19.

Despite the availability of these recommendations in the media, the surge of requests for information and advice could not be entirely managed by the health authorities through public health care helplines and family doctors. Indeed, a large part of the population tried to find appropriate answers to their questions on the web [4].

Online decision support tools have been used in the past, such as during the $\mathrm{A} / \mathrm{H} 1 \mathrm{~N} 1 \mathrm{pdm} 09$ pandemic [5], to meet the information needs of the public at large, improve measures to prevent transmission, and support the management of symptomatic cases. During the COVID-19 pandemic, online decision support systems (DSS) and artificial intelligence chatbots have also been used for digital triage and self-diagnosis and have been shown to be accurate in identifying suspected COVID-19 cases and efficient in decompressing the pressure placed on emergency rooms and helplines [6-12]. On the other hand, the adoption of digital tools encounters barriers and limitations intrinsic to their novelty, the digital divide, and other local circumstances [6,9].

To provide clinical and public health recommendations in line with the evolving guidelines issued by the Italian Ministry of Health, in February 2020, Paginemediche, a digital Italian health tech startup, developed a noncommercial, online DSS available in Italian [13]. The system was designed as a simple algorithm for assisting individuals manage their potential exposure to COVID-19 and has a chat user interface. The system was available on several web pages including landing pages of the regional health systems and was advertised through social media. A link to the online DSS was also hosted on the home page of the Regional Health Authorities of Lombardy and Campania and of Trento autonomous province.

Digital platforms collecting self-reported information have been shown to provide complementary information to traditional epidemiological surveillance [14]. In particular, it has been reported that patterns of use of online tools for COVID-19 self-triage may be considered a proxy of confirmed cases reported to national surveillance systems and may provide early signals of COVID-19 cases [15].

As our online DSS was available on a national scale from the early phase of the pandemic and for a time period of 1 year, we examined it as a potential data source for digital surveillance of COVID-19 in Italy. Our hypothesis was that trends in the use of the online DSS may precede those observed in the national surveillance system, as symptomatic individuals and those who have been in contact with a COVID-19 case may access the online DSS before undergoing a laboratory test for confirmation.

The aim of the present study was to describe the general characteristics of users of the online DSS; compare the trends in online DSS sessions with those of COVID-19 cases reported by the national health surveillance system in Italy, from February 2020 to March 2021; and study the time lag between the online DSS and the surveillance system data.

\section{Methods}

Our online DSS was developed as a web-based algorithm to triage users with COVID-19-compatible symptoms or with a history of contact with a confirmed COVID-19 case and to provide recommendations on appropriate management (including testing), according to guidelines from the Italian Ministry of Health [16]. Anonymous access to the system is open to any user, and interaction is based on a chat interface. The system is available on the Paginemediche website [13].

Briefly, at the beginning of the session, the system collects information on age group and place of residence. Then, the user is asked to select the reason for accessing the system from among the following: (1) evaluation of symptoms, (2) contact with a confirmed COVID-19 case, (3) notification received from the national contact tracing app [17], (4) positive swab for COVID-19. The system then proceeds by matching the information provided by the users with national guidelines and provides tailored recommendations, such as asking for immediate medical support, contacting the family doctor, performing a diagnostic test, or applying nonpharmaceutical measures such as isolation and self-quarantine.

As the purpose of this study was to test if access to the online DSS preceded (and therefore was able to predict) national surveillance system trends, we restricted our analysis to users accessing the system for the evaluation of symptoms (SYM) or for contact with a confirmed COVID-19 case (CON).

We analyzed the information on users' age and location and the symptoms reported by age group using descriptive statistics. We then explored the temporal trends in SYM and CON sessions, and we compared them with trends in COVID-19 cases officially reported by the Italian COVID-19 national surveillance system, which is based on the data from laboratory-confirmed 
SARS-CoV-2 infections provided daily by regional health authorities to the National Health Institute. To perform the comparison, we first used moving averages to display the curves.

$$
M A_{k}=\frac{1}{k} \sum_{i=n-k+1}^{n} p_{i}
$$

where $p_{i}$ are data points.

$\mathrm{K}$ was set at a value of 7 , as data from the national surveillance system were published weekly.

We then scaled each time series in a range between 0 and 1 , applying the following formula:

$$
x_{\text {scaled }}=\frac{x-\min (x)}{\max (x)-\min (x)}
$$

To calculate the distance between the time series of cases reported through the national surveillance system and those of SYM and CON, we used the dynamic time warping algorithm (DTW) [18,19]. DTW measures similarity between 2 temporal sequences, which may vary in speed. The sequences are "warped" nonlinearly in the time dimension to determine a measure of their similarity, independent of nonlinear variations in time.

To confirm the hypothesis that the online DSS trends anticipated those of reported COVID-19 cases, we first applied Symbolic Aggregate approXimation (SAX) encoding [20] to the time series in 1-week periods. Then, we calculated the Hamming distance [21,22] between the SAX strings. SAX transforms a time series into a sequence of symbols that represents a range of values, allowing the application of the dimensionality reduction on the time series $[20,23]$. SAX encoding was chosen as it allows dimensionality/numerosity reduction of the time series and as it is relatively easy to understand and compute. The dimensionality reduction was based on Piecewise Aggregate Approximation [20,22]. To verify that the online DSS anticipated the trends observed in notified cases, we shifted its time series 1 week ahead, and we measured the improvement in the Hamming distance [24]. The 1-week shift was chosen empirically based on the knowledge that the average incubation period is 4 days [25], the median time to diagnosis is 5.85 days [26], and most Italian laboratories have been providing results for PCR tests $24-48$ hours after the test. Other authors have chosen the same time shift for modelling purposes [27].

This study includes data collected by the online DSS from February 25, 2020 to March 15, 2021 and the corresponding figures of COVID-19 cases in Italy published by the national
COVID-19 surveillance system in their open access repository [28].

The statistical analysis was conducted using Python 3.8.5.

Considering the nature of the analysis, the current study did not require approval by the local ethics committee according to current legislation. However, a notification including the study characteristics was sent to the Bambino Gesù Ethical Committee.

\section{Results}

We recorded a total of 75,557 sessions in the online DSS during the study period. Among them, 65,207 were SYM, and 19,062 were CON. Among the 65,207 sessions of users with symptoms, 8692 stated they also had contact with a COVID-19-positive individual.

Age was missing in nearly $32 \%(24,084 / 75,557,31.87 \%)$ of the sessions, and place of residence was missing in $28.27 \%$ $(21,360 / 75,557)$, probably because the input of information about age and place of residence was not compulsory in the first 2 months of activity in the online DSS. Among the 51,473 sessions for which age was recorded, the majority $(40,923 / 51,473,79.50 \%)$ were by adults in the 19-64-year-old age group: $19-24$ years old, 4830/51,473, 9.38\%; 25-34 years old, 9054/51,473, 17.59\%; 35-44 years old, 10,383/51,473, 20.17\%; 45-54 years old, 9852/51,473, 19.14\%; 55-64 years old, 6804/51,473, $13.22 \%$. Users 65 years old and older represented $12.89 \%(6636 / 51,473)$ of the sessions $(65-74$ years, $4339 / 51,473,8.43 \%$; $\geq 75$ years, $2297 / 51,473,4.46 \%)$, and $7.60 \%(3914 / 51,473)$ of the sessions were by users $\leq 18$ years of age. Sessions were established from all Italian regions, at a proportion between 0.3 and 0.6 per 1000 inhabitants. The number of sessions by region are described in Figure 1. Three regions were more represented, reflecting more active local endorsement by regional authorities: 2 in Northern Italy, namely Lombardy and the Trento autonomous province, and 1 in Southern Italy, namely Campania.

Symptoms reported by online DSS users by age group are illustrated in Table 1.

Symptoms reported by users varied with age. In all age groups, fever was the most reported symptom, although the proportion of patients reporting fever was higher in younger individuals and decreased with age. A similar trend was observed for respiratory symptoms, such as cold and sore throat. Among other symptoms, myalgia, low blood pressure, and dizziness were most frequently reported among people belonging to older age groups. 
Figure 1. Number of sessions by the number of residents in each region.

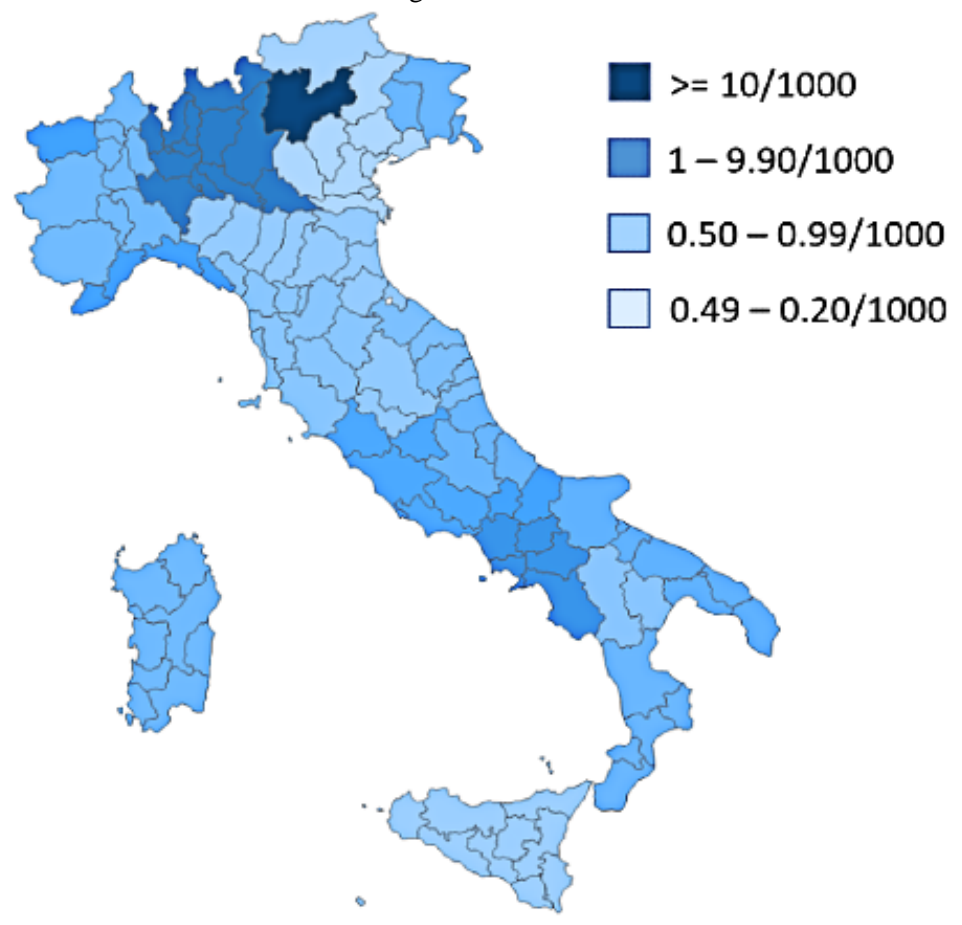

Table 1. Distribution of symptoms reported in each session by age group $(n=51,473)$.

\begin{tabular}{lllll}
\hline Symptom & Age (years), $\mathrm{n}(\%)$ & & & \\
& $0-18(\mathrm{n}=3914)$ & $19-64(\mathrm{n}=40,923)$ & $65-74(\mathrm{n}=4339)$ & $\geq 75(\mathrm{n}=2297)$ \\
\hline Fever & $1912(48.85)$ & $12,896(31.51)$ & $993(22.89)$ & $625(27.21)$ \\
Cold & $1489(38.04)$ & $9584(23.42)$ & $801(18.46)$ & $406(17.68)$ \\
Sore throat & $1027(26.24)$ & $10,264(25.08)$ & $628(14.47)$ & $305(13.28)$ \\
Cough & $881(22.51)$ & $8540(20.87)$ & $831(19.15)$ & $441(19.20)$ \\
Headache & $437(11.17)$ & $4870(11.90)$ & $280(6.45)$ & $82(3.57)$ \\
Myalgia & $262(6.69)$ & $4947(12.09)$ & $643(14.82)$ & $284(12.36)$ \\
Dispnea & $381(9.73)$ & $4296(10.50)$ & $362(8.34)$ & $314(13.67)$ \\
Low blood pressure & $93(2.38)$ & $2612(6.38)$ & $611(14.08)$ & $322(14.02)$ \\
Dizziness & $301(7.69)$ & $2504(6.12)$ & $298(6.87)$ & $251(10.93)$ \\
Drowsiness & $214(5.47)$ & $2195(5.36)$ & $269(6.20)$ & $220(9.58)$ \\
Tachycardia & $260(6.64)$ & $2043(4.99)$ & $265(6.11)$ & $138(6.01)$ \\
Nausea & $285(7.28)$ & $1947(4.76)$ & $157(3.62)$ & $61(2.66)$ \\
\hline
\end{tabular}

Figure 2 shows the numbers of SYM and CON sessions over time and the number of confirmed COVID-19 cases officially reported to the national surveillance system.

The highest number of sessions was recorded in the early phases of the pandemic, when COVID-19-confirmed cases started to increase very quickly.

A less pronounced peak was observed in the number of CON sessions in the same period. A second peak, both in SYM and CON sessions, was observed during a large second wave of COVID-19 cases, which, in Italy, started in October 2020. Finally, a third peak was observed in March 2021, when Italy experienced a third surge of cases. Figure 3 shows the trends in SYM sessions, CON sessions, and COVID-19 cases as moving averages in 1-week periods and scaling values between 0 and 1.

The 3 time series in the entire time period had a similar trend, independently from the amplitude of the peaks, although an increase in SYM and CON anticipated the trend in confirmed COVID-19 cases by approximately 1 week. The distance between SYM and notified COVID-19 cases calculated through DTW (the lower the distance, the higher the similarity between the curves) was 93.72, while that between CON and notified COVID-19 cases was 61.23 .

As CON was more consistent with the trend in confirmed cases than SYM, we tested the hypothesis that this series anticipated confirmed cases by 1 week. After applying SAX encoding, we 
measured the Hamming distance between the time series of confirmed COVID-19 cases and the CON series (1) as recorded by the system and (2) shifted by 1 week. After applying the 1 -week shift, the Hamming distance improved from 0.49 to
0.46 . We also repeated the analysis restricting the time window to the time period between July 2020 and December 2020. The corresponding Hamming distance was 0.16 before shifting the time series and improved to 0.08 after the time shift (Figure 4).

Figure 2. Weekly number of COVID-19 confirmed cases (red), SYM (symptomatic users) sessions (yellow), and CON (users with contact with a COVID-19 case; green) sessions between February 2020 and March 2021.

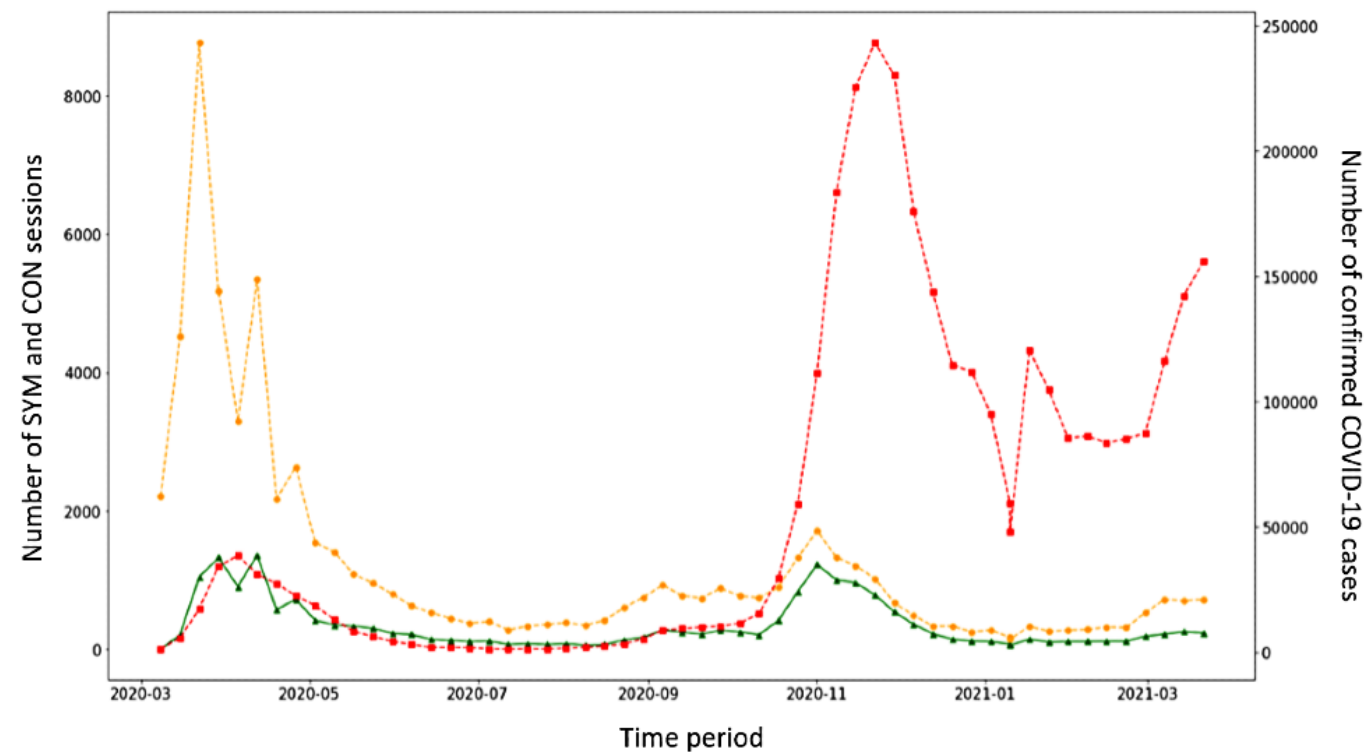

Figure 3. Time trends in SYM (symptomatic users) sessions (yellow), CON (users with contact with a COVID-19 case) sessions (green), and COVID-19 cases (red). Values are illustrated as rolling means in 1-week periods and scaled to values between 0 and 1.

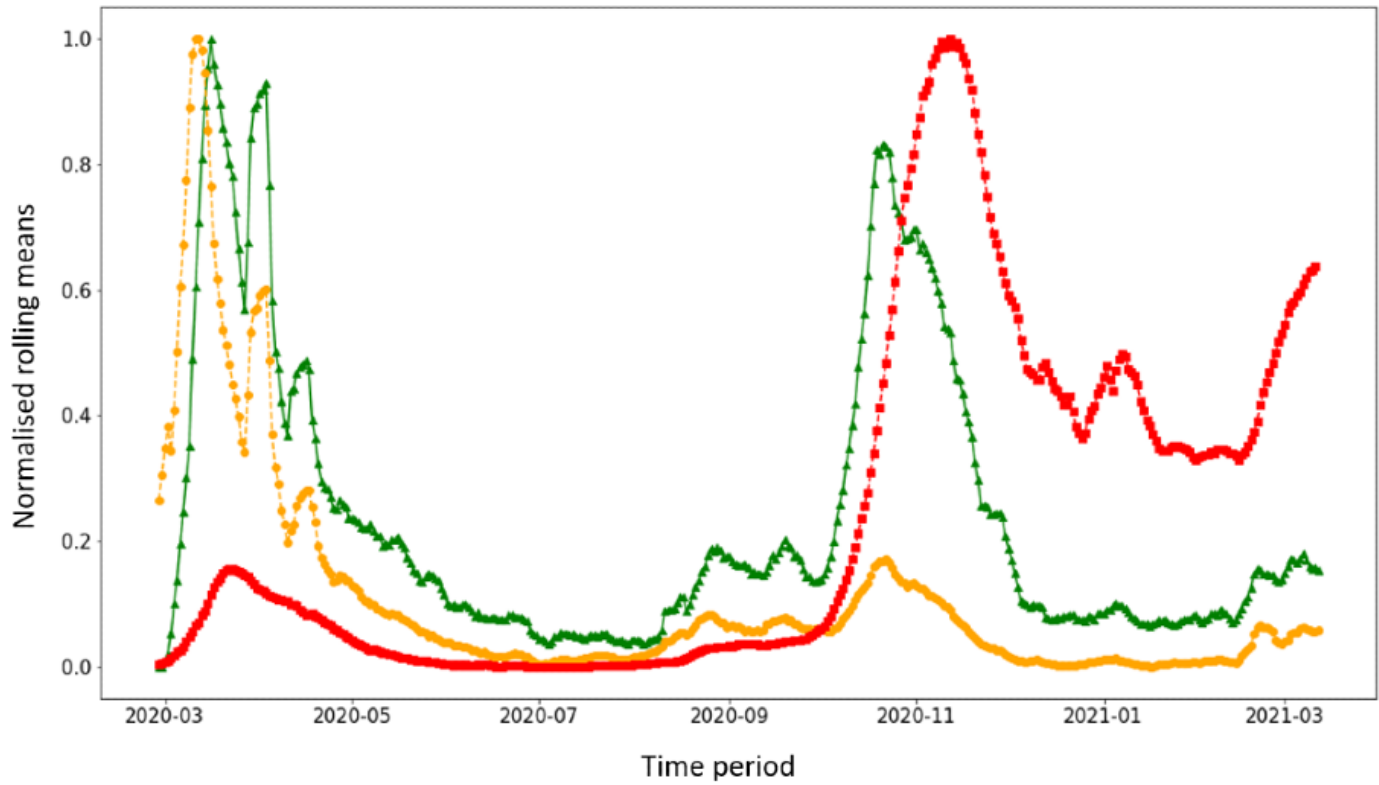


Figure 4. Time trends in CON (users with contact with a COVID-19 case) sessions and notified COVID-19 cases, Italy, July 2020 to December 2020. Values are illustrated as rolling means and scaled from 0 to 1 . The CON time series is shifted 7 days ahead.

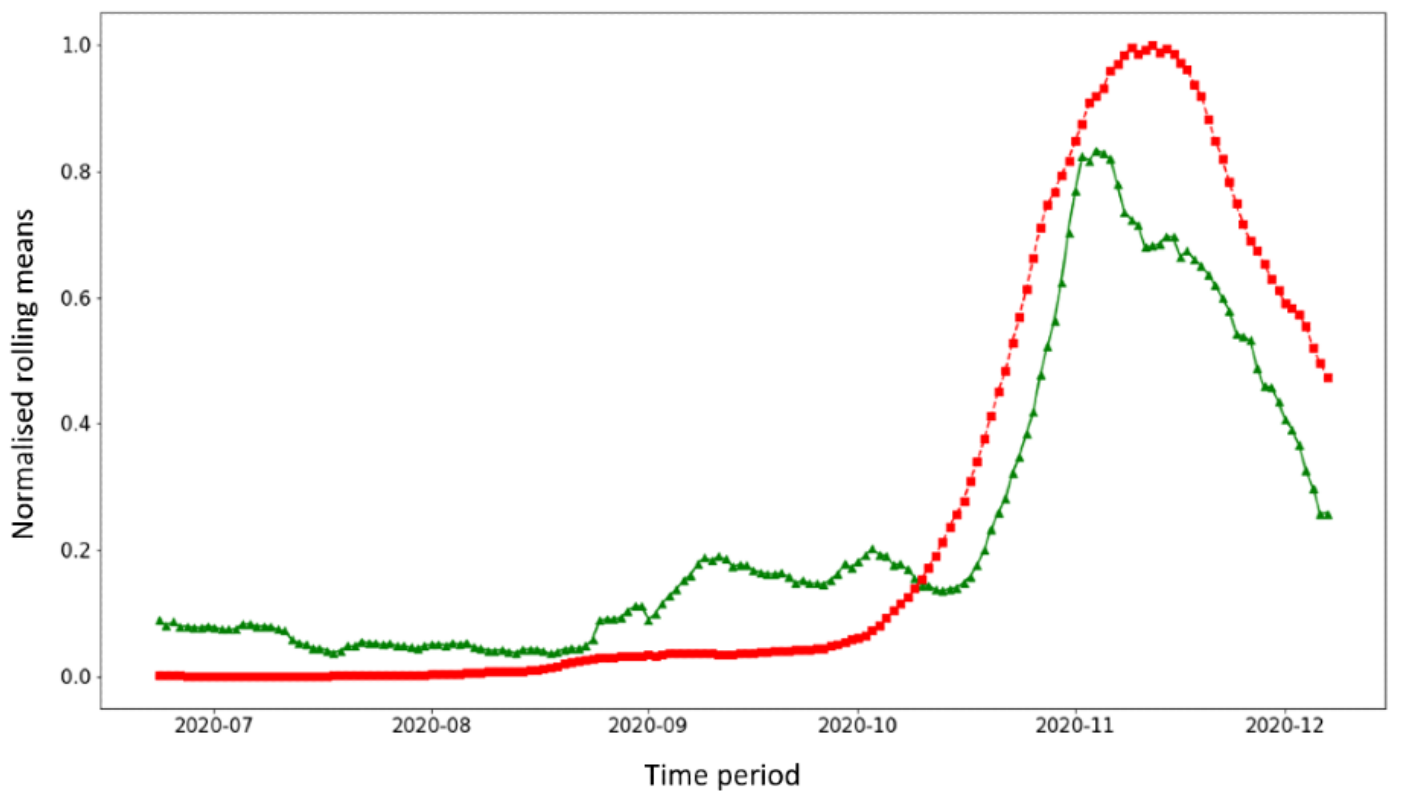

\section{Discussion}

In this study, we showed that users of a national online DSS reporting contact with a COVID-19 case anticipated trends observed by the Italian national surveillance system by 1 week.

Surveillance systems for infectious diseases frequently suffer from lack of timeliness and difficulties in case finding that may delay the implementation of effective, data-based preventative strategies [29,30]. Digital surveillance has been indicated as a potential supplement to traditional surveillance due to the fact that web and social media users may spontaneously leave online traces related to their health conditions, which can be detected by digital surveillance systems in a timely manner [31].

Classically, digital traces for surveillance are classified as either based on the public's demand of information (eg, volume of searches on Google) or on information supplied by internet and social media users (eg, tweets reporting symptoms of a specific disease) [32]. Indeed, several attempts have been made to track and interpret the signals from queries on search engines or from keywords or symptoms reported on social media to promptly recognize the emergence of infectious diseases, with mixed success [33]. As a matter of fact, trends in queries on search engines are strongly affected by media coverage that may not accurately reflect the epidemic trends [34].

With this study, we explored the potential of a digital surveillance system based on a mixed demand-supply method. Users accessed our online DSS with a specific request for information and were invited to provide specific data on their condition through a structured framework. The algorithm guided the user through predefined questions based on national guidelines for COVID-19 prevention and contact management, with the aim to provide tailored, actionable recommendations.

Our results show that temporal trends in the number of sessions on an online COVID-19 DSS dedicated to the general public may precede the trend in confirmed COVID-19 cases obtained through the national surveillance system by 7 days. We found a high correlation between the trend in sessions by users accessing the online DSS (with either a COVID-19 contact or symptoms) and the number of notified COVID-19 cases, documented by the short distance between the trends as estimated with DTW. However, the trends in sessions by users who had contact with COVID-19 cases predicted the trend in COVID-19 cases better than sessions by users with symptoms. This observation is plausible as, on the one hand, recognition of contact with someone with COVID-19 usually precedes the onset of symptoms, and, on the other hand, contact with a COVID-19 case can be considered as a more specific proxy of COVID-19, as the clinical picture of COVID-19 may overlap with that caused by other diseases. The correlation between sessions by users with a COVID-19 contact and confirmed cases greatly improved when the analysis was restricted to the central phase of the pandemic, from July 2020 to December 2020. In this time period, Italy experienced the highest surge of COVID-19 cases.

To our knowledge, only one other study attempted to use sessions in an online DSS as a proxy for case notification [15], showing lead times of 3 days in China and 19 days in the United States. Although the recognition of contact with a COVID-19 case may be delayed, our estimate of a 7-day lead time from users with contact with a COVID-19 case is consistent with the incubation of COVID-19 [26] and the time needed to perform a diagnostic test and obtain results.

These results show that the utility of an online DSS dedicated to the general public may go beyond its original purpose of guiding the public through recommendations for the management of cases or contacts.

First, these systems may generate early warning signals that may inform early interventions (eg, tailored restriction measures), as is desirable during the current pandemic. Data from our system may be particularly valuable in predicting large and rapid increases in COVID-19 cases, as shown by the higher 
correlation between the trend in contacts and national notifications.

Second, the high correlation estimates obtained through DTW support the inclusion of online DSS-based data in the development of prediction models, which could be useful to anticipate an increase in cases as well as to predict the timing of a reduction in cases. This would support national or regional institutions in more finely programming (timing, intensity, logistics, economic impact) the restrictive measures to contain the epidemic.

Digital data have already been used in prediction models for influenza. Through the analysis of the volume of online searches for flu-related terms, Google Flutrends offered a forecast of the trend in influenza-like illness cases in the United States [35], and a similar approach has recently been used in the Netherlands [36]. Data for participatory surveillance have also been integrated with classic surveillance data [37]. Although prediction models including participatory surveillance data can suffer from a lack of representativeness of the population sample, modeling and simulation can help control this bias [38].

In a recent study, Kogan et al [39] attempted to combine and harmonize different digital data streams to predict COVID-19 trends. They included Google Trends, Twitter, UpToDate, and mobility data, but no data from online DSSs were taken into account, which could have potentially improved the performance of the model.

For the purpose of informing prediction models with digital surveillance data, online DSS data would benefit from integration with data from contact tracing apps. Contact tracing apps have been developed in several countries, including Italy, to support public health measures for COVID-19 containment. These digital tools send a notification of exposure to a COVID-19-positive case to contacts and recommend preventative actions. However, data about the number of contacts and other personal characteristics remain stored in personal smartphones and cannot be used as a data source for surveillance. Moreover, although Italy has been considered as one of the countries with the highest acceptance of apps for contact tracing [40], only $20 \%$ of the general population actually downloaded the app as of March 2021 [41].

In this article, we report national data obtained through our online DSS. However, it should be noted that a very high user rate has been recorded in the Lombardia and Campania regions and in the autonomous province of Trento. The potential of predicting actual COVID-19 trends was more accurate in areas with a higher coverage of the system. In these regions, the online DSS had been actively promoted by local health institutions. The involvement of health authorities and the promotion of these systems by digital marketing through online social media are likely to increase the use of these digital tools, underlying their value in providing tailored recommendations to individuals. Stronger involvement of health authorities, by increasing participation and accuracy, would also allow us to develop prediction models on a regional — rather than on a national basis.
This study has several strengths. First, data were available over a 12-month period on a national scale. We also found that the online DSS was accessed from all Italian regions, which was reassuring for the representativeness of the data sample. The system was also open to any user, as there was no age restriction for accessing the system.

The study also has obvious limitations. The most important is that we could not validate information provided by users, as expected from any anonymous online tool. Moreover, the number of sessions in an online DSS like ours may be affected by different factors that may increase the background noise and make the interpretation of trends difficult. Some of the fluctuations observed in the use of the online DSS may reflect a different perception of risk during different phases of the pandemic. Indeed, infodemics may affect perception of risk of infection that may result in an increase in online searches for information by the general public [42]. We cannot exclude that users' access to our online DSS might have been biased by different trends in interest by the general public, possibly affected by different media coverage of the pandemic throughout the study period. Nevertheless, the high correlation between our access data and the epidemic curve suggests that the variation of users' interest and, subsequently, of their online searches has not had a major impact on the use of our online DSS, which seems to have been primarily affected by the disease incidence. Nevertheless, in order to better validate a system like the one proposed in our study, future research should better estimate the impact of online searches on access to the online DSS, possibly correcting the analysis for the volume of searches based on keywords related to the epidemic.

Moreover, especially in the early phase of the pandemic, users may have tried to simulate different scenarios to better understand what to expect in case of symptoms or contact with a COVID-19 case. As a matter of fact, when restricting the analysis by excluding the earliest phase of the pandemic, the consistency of the online DSS time series with confirmed COVID-19 cases was higher. Furthermore, we did not collect socioeconomic information, and we could not attempt to adjust our analyses for confounders. Finally, all data streams from digital systems may suffer from selection bias due to the digital divide [43]. As a matter of fact, only $13 \%$ of the sessions were by individuals over the age of 65 years, which was the age group mostly affected in the first phase of the pandemic. Therefore, we should consider that this age group may have not been well represented in the online DSS sessions, and this might have affected the accuracy of our prediction. Nevertheless, we were not interested in estimating the incidence by age group based on the online DSS data, as our main objective was to study the trends in access to the online DSS and their relationship with official surveillance data. The high number of sessions recorded by any age group and from any region and their consistency over time with the trend in notified cases are reassuring that potential major biases are not present.

In conclusion, as online DSSs like the one described in this study are widely available and may be completely anonymous, they may represent a significant source of data to inform public health actions, may supplement other epidemiological 
information collected through other means, and may help to develop more accurate prediction models for pandemic trends.

Public health strategies for immunization and containment are usually based on incidence of preventable diseases and vaccine coverage, which carry some latency due to the notification systems. Despite the observed limitations, prediction models based on systems like the one described in our study may anticipate other epidemiological signals of epidemic surge and, when integrated with official surveillance data, can support timely decisions for mitigating the pandemic spread.
Digital tools for managing COVID-19 not only have epidemiological implications but also may be extremely helpful in disseminating appropriate recommendations to the general population in a universal strategy.

Although it remains to be assessed how frequently recommendations provided are actually followed by users and selection bias should be better understood and addressed, these tools represent a potential data source for public health that may help, in combination with others, to expeditiously implement containment strategies.

\section{Acknowledgments}

We thank Kiersten Miller for revising the English manuscript and Tina Dell'Armi for providing support for data management and analysis.

\section{Authors' Contributions}

AET conceived the study and drafted the manuscript. RA conceived the study and contributed to interpretation of results. FG drafted the manuscript and contributed to interpretation of results. CR contributed to interpretation of results and revised the manuscript. EU and AS contributed to data extraction, management, and analysis. NP and IC performed the statistical analysis.

\section{Conflicts of Interest}

EU, AS, RA, and NP work for Paginemediche and Healthware Group, which were involved in the development of the online DSS included on the Paginemediche website. CR received grants for participating in Advisory Boards. The other authors declare no conflicts of interest.

\section{References}

1. WHO Coronavirus (COVID-19) Dashboard. World Health Organization. URL: https://covid19.who.int [accessed 2021-08-01]

2. Spiteri G, Fielding J, Diercke M, Campese C, Enouf V, Gaymard A, et al. First cases of coronavirus disease 2019 (COVID-19) in the WHO European Region, 24 January to 21 February 2020. Euro Surveill 2020 Mar;25(9):1 [FREE Full text] [doi: 10.2807/1560-7917.ES.2020.25.9.2000178] [Medline: $\underline{32156327]}$

3. Onder G, Rezza G, Brusaferro S. Case-Fatality Rate and Characteristics of Patients Dying in Relation to COVID-19 in Italy. JAMA 2020 May 12;323(18):1775-1776. [doi: 10.1001/jama.2020.4683] [Medline: 32203977]

4. Rovetta A, Bhagavathula AS. COVID-19-Related Web Search Behaviors and Infodemic Attitudes in Italy: Infodemiological Study. JMIR Public Health Surveill 2020 May 05;6(2):e19374 [FREE Full text] [doi: 10.2196/19374] [Medline: 32338613]

5. Spaulding AB, Radi D, Macleod H, Lynfield R, Larson M, Hyduke T, et al. Design and implementation of a statewide influenza nurse triage line in response to pandemic H1N1 influenza. Public Health Rep 2012;127(5):532-540 [FREE Full text] [doi: 10.1177/003335491212700509] [Medline: 22942472]

6. Lai L, Wittbold KA, Dadabhoy FZ, Sato R, Landman AB, Schwamm LH, et al. Digital triage: Novel strategies for population health management in response to the COVID-19 pandemic. Healthc (Amst) 2020 Dec;8(4):100493 [FREE Full text] [doi: 10.1016/j.hjdsi.2020.100493] [Medline: $\underline{33129176]}$

7. Hautz WE, Exadaktylos A, Sauter TC. Online forward triage during the COVID-19 outbreak. Emerg Med J 2020 Dec 11:1 [FREE Full text] [doi: 10.1136/emermed-2020-209792] [Medline: 33310732]

8. Haase CB, Bearman M, Brodersen J, Hoeyer K, Risor T. 'You should see a doctor', said the robot: Reflections on a digital diagnostic device in a pandemic age. Scand J Public Health 2021 Feb;49(1):33-36 [FREE Full text] [doi: 10.1177/1403494820980268] [Medline: 33339468]

9. Fan X, Chao D, Zhang Z, Wang D, Li X, Tian F. Utilization of Self-Diagnosis Health Chatbots in Real-World Settings: Case Study. J Med Internet Res 2021 Jan 06;23(1):e19928 [FREE Full text] [doi: 10.2196/19928] [Medline: 33404508]

10. Judson TJ, Odisho AY, Young JJ, Bigazzi O, Steuer D, Gonzales R, et al. Implementation of a digital chatbot to screen health system employees during the COVID-19 pandemic. J Am Med Inform Assoc 2020 Jul 01;27(9):1450-1455 [FREE Full text] [doi: 10.1093/jamia/ocaa130] [Medline: 32531066]

11. Galmiche S, Rahbe E, Fontanet A, Dinh A, Bénézit F, Lescure F, et al. Implementation of a Self-Triage Web Application for Suspected COVID-19 and Its Impact on Emergency Call Centers: Observational Study. J Med Internet Res 2020 Nov 23;22(11):e22924 [FREE Full text] [doi: 10.2196/22924] [Medline: 33147165]

12. Martin A, Nateqi J, Gruarin S, Munsch N, Abdarahmane I, Zobel M, et al. An artificial intelligence-based first-line defence against COVID-19: digitally screening citizens for risks via a chatbot. Sci Rep 2020 Nov 04;10(1):19012 [FREE Full text] [doi: 10.1038/s41598-020-75912-x] [Medline: 33149198]

13. Paginemediche: la piattaforma che connette medici e pazienti. URL: https://www.paginemediche.it/ [accessed 2021-08-01] 
14. Menni C, Valdes AM, Freidin MB, Sudre CH, Nguyen LH, Drew DA, et al. Real-time tracking of self-reported symptoms to predict potential COVID-19. Nat Med 2020 Jul;26(7):1037-1040 [FREE Full text] [doi: 10.1038/s41591-020-0916-2] [Medline: 32393804$]$

15. Hswen Y, Brownstein JS, Xu X, Yom-Tov E. Early detection of COVID-19 in China and the USA: summary of the implementation of a digital decision-support and disease surveillance tool. BMJ Open 2020 Dec 10;10(12):e041004 [FREE Full text] [doi: 10.1136/bmjopen-2020-041004] [Medline: $\underline{33303453]}$

16. Cosa sapere su test, tracciamento, quarantena. Ministero della Salute. URL: https://www.salute.gov.it/portale/ nuovocoronavirus/dettaglioFaqNuovoCoronavirus.jsp?lingua=italiano\&id=244 [accessed 2021-08-01]

17. immuni-app / immuni-documentation. GitHub. URL: https://github.com/immuni-app/immuni-documentation [accessed 2021-08-01]

18. Sakoe H, Chiba S. Dynamic programming algorithm optimization for spoken word recognition. IEEE Trans. Acoust., Speech, Signal Process 1978 Feb;26(1):43-49. [doi: 10.1109/TASSP.1978.1163055]

19. Salvador S, Chan P. Toward accurate dynamic time warping in linear time and space. IDA 2007 Oct 10;11(5):561-580. [doi: $10.3233 /$ ida-2007-11508]

20. Lin J, Keogh E, Wei L, Lonardi S. Experiencing SAX: a novel symbolic representation of time series. Data Min Knowl Disc 2007 Apr 3;15(2):107-144. [doi: 10.1007/s10618-007-0064-z]

21. Hamming RW. Error Detecting and Error Correcting Codes. Bell System Technical Journal 1950;29(2):147-160 [FREE Full text] [doi: 10.1002/j.1538-7305.1950.tb00463.x]

22. Keogh E, Chakrabarti K, Pazzani M, Mehrotra S. Dimensionality Reduction for Fast Similarity Search in Large Time Series Databases. Knowledge and Information Systems 2001 Aug;3(3):263-286. [doi: 10.1007/p100011669]

23. Lin J, Keogh E, Lonardi S, Chiu B. A symbolic representation of time series, with implications for streaming algorithms. 2003 Presented at: 8th ACM SIGMOD workshop on Research issues in data mining and knowledge discovery; June 13, 2003; San Diego, CA. [doi: 10.1145/882082.882086]

24. scipy.spatial.distance.hamming. SciPy. URL: https://docs.scipy.org/doc/scipy/reference/generated/scipy.spatial.distance. hamming.html [accessed 2021-08-01]

25. Zardini A, Galli M, Tirani M, Cereda D, Manica M, Trentini F, et al. A quantitative assessment of epidemiological parameters to model COVID- 19 burden. Cornell University. 2021 Mar 29. URL: http://arxiv.org/abs/2103.15780 [accessed 2021-08-01]

26. Ejima K, Kim KS, Ludema C, Bento AI, Iwanami S, Fujita Y, et al. Estimation of the incubation period of COVID-19 using viral load data. Epidemics 2021 Jun;35:100454 [FREE Full text] [doi: 10.1016/j.epidem.2021.100454] [Medline: 33773195]

27. Turk PJ, Tran TP, Rose GA, McWilliams A. A predictive internet-based model for COVID-19 hospitalization census. Sci Rep 2021 Mar 03;11(1):5106 [FREE Full text] [doi: 10.1038/s41598-021-84091-2] [Medline: 33658529]

28. pcm-dpc / COVID-19. GitHub. URL: https://github.com/pcm-dpc/COVID-19 [accessed 2021-08-01]

29. Jajosky RA, Groseclose SL. Evaluation of reporting timeliness of public health surveillance systems for infectious diseases. BMC Public Health 2004 Jul 26;4:29 [FREE Full text] [doi: 10.1186/1471-2458-4-29] [Medline: 15274746]

30. Swaan C, van den Broek A, Kretzschmar M, Richardus JH. Timeliness of notification systems for infectious diseases: A systematic literature review. PLoS One 2018 Jun 14;13(6):e0198845 [FREE Full text] [doi: 10.1371/journal.pone.0198845] [Medline: 29902216]

31. Mavragani A. Tracking COVID-19 in Europe: Infodemiology Approach. JMIR Public Health Surveill 2020 Apr 20;6(2):e18941 [FREE Full text] [doi: 10.2196/18941] [Medline: 32250957]

32. Eysenbach G. Infodemiology and infoveillance: framework for an emerging set of public health informatics methods to analyze search, communication and publication behavior on the Internet. J Med Internet Res 2009 Mar 27;11(1):e11 [FREE Full text] [doi: 10.2196/jmir.1157] [Medline: 19329408]

33. Lazer D, Kennedy R, King G, Vespignani A. Big data. The parable of Google Flu: traps in big data analysis. Science 2014 Mar 14;343(6176):1203-1205. [doi: 10.1126/science.1248506] [Medline: 24626916]

34. Sousa-Pinto B, Anto A, Czarlewski W, Anto JM, Fonseca JA, Bousquet J. Assessment of the Impact of Media Coverage on COVID-19-Related Google Trends Data: Infodemiology Study. J Med Internet Res 2020 Aug 10;22(8):e19611 [FREE Full text] [doi: 10.2196/19611] [Medline: 32530816]

35. Ginsberg J, Mohebbi MH, Patel RS, Brammer L, Smolinski MS, Brilliant L. Detecting influenza epidemics using search engine query data. Nature 2009 Feb 19;457(7232):1012-1014. [doi: 10.1038/nature07634] [Medline: 19020500]

36. Schneider PP, van Gool CJ, Spreeuwenberg P, Hooiveld M, Donker GA, Barnett DJ, et al. Using web search queries to monitor influenza-like illness: an exploratory retrospective analysis, Netherlands, 2017/18 influenza season. Euro Surveill 2020 May;25(21):1 [FREE Full text] [doi: 10.2807/1560-7917.ES.2020.25.21.1900221] [Medline: 32489174$]$

37. Guerrisi C, Turbelin C, Blanchon T, Hanslik T, Bonmarin I, Levy-Bruhl D, et al. Participatory Syndromic Surveillance of Influenza in Europe. J Infect Dis 2016 Dec 01;214(suppl_4):S386-S392. [doi: 10.1093/infdis/jiw280] [Medline: 28830105]

38. Brownstein JS, Chu S, Marathe A, Marathe MV, Nguyen AT, Paolotti D, et al. Combining Participatory Influenza Surveillance with Modeling and Forecasting: Three Alternative Approaches. JMIR Public Health Surveill 2017 Nov 01;3(4):e83 [FREE Full text] [doi: 10.2196/publichealth.7344] [Medline: 29092812] 
39. Kogan NE, Clemente L, Liautaud P, Kaashoek J, Link NB, Nguyen AT, et al. An early warning approach to monitor COVID-19 activity with multiple digital traces in near real time. Sci Adv 2021 Mar;7(10):1 [FREE Full text] [doi: 10.1126/sciadv.abd6989] [Medline: 33674304]

40. Altmann S, Milsom L, Zillessen H, Blasone R, Gerdon F, Bach R, et al. Acceptability of App-Based Contact Tracing for COVID-19: Cross-Country Survey Study. JMIR Mhealth Uhealth 2020 Aug 28;8(8):e19857 [FREE Full text] [doi: 10.2196/19857] [Medline: 32759102]

41. Immuni. URL: https://www.immuni.italia.it/ [accessed 2021-08-01]

42. Tangcharoensathien V, Calleja N, Nguyen T, Purnat T, D'Agostino M, Garcia-Saiso S, et al. Framework for Managing the COVID-19 Infodemic: Methods and Results of an Online, Crowdsourced WHO Technical Consultation. J Med Internet Res 2020 Jun 26;22(6):e19659 [FREE Full text] [doi: 10.2196/19659] [Medline: 32558655]

43. Ramsetty A, Adams C. Impact of the digital divide in the age of COVID-19. J Am Med Inform Assoc 2020 Jul 01;27(7):1147-1148 [FREE Full text] [doi: 10.1093/jamia/ocaa078] [Medline: 32343813]

\section{Abbreviations}

CON: users accessing the system because of contact with a confirmed COVID-19 case

DSS: decision support system

DTW: dynamic time warping

SAX: Symbolic Aggregate approXimation

SYM: users accessing the system for the evaluation of symptoms

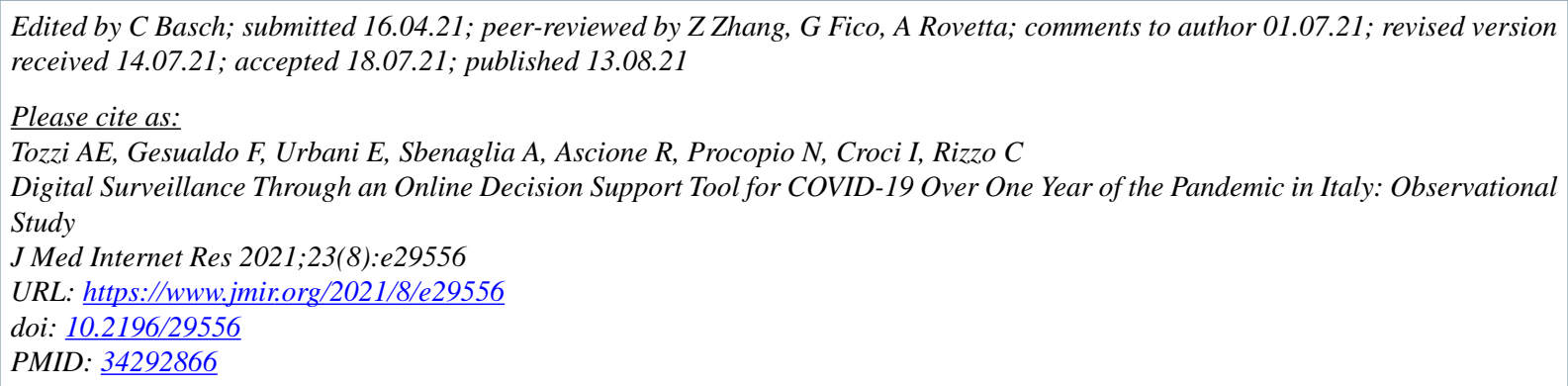

(C)Alberto Eugenio Tozzi, Francesco Gesualdo, Emanuele Urbani, Alessandro Sbenaglia, Roberto Ascione, Nicola Procopio, Ileana Croci, Caterina Rizzo. Originally published in the Journal of Medical Internet Research (https://www.jmir.org), 13.08.2021. This is an open-access article distributed under the terms of the Creative Commons Attribution License (https://creativecommons.org/licenses/by/4.0/), which permits unrestricted use, distribution, and reproduction in any medium, provided the original work, first published in the Journal of Medical Internet Research, is properly cited. The complete bibliographic information, a link to the original publication on https://www.jmir.org/, as well as this copyright and license information must be included. 